\title{
Compressive sensing imaging for general synthetic aperture radar echo model based on Maxwell's equations
}

\author{
Bing Sun ${ }^{1,2}$, Yufeng Cao ${ }^{2,3}$, Jie Chen ${ }^{1 *}$, Chunsheng $\mathrm{Li}^{1}$ and Zhijun Qiao ${ }^{2}$
}

\begin{abstract}
A general echo model is derived for the synthetic aperture radar (SAR) imaging with high resolution based on the scalar form of Maxwell's equations. After analyzing the relationship between the general echo model in frequency domain and the existing model in time domain, a compressive sensing (CS) matrix is constructed from random partial Fourier matrices for processing the range CS SAR imaging. Simulations validate the orthogonality of the proposed CS matrix and the feasibility of CS SAR imaging based on the general echo model.
\end{abstract}

Keywords: Compressive sensing; General echo model; Maxwell's equations; Synthetic aperture radar

\section{Introduction}

Synthetic aperture radar (SAR) has been in development for more than 60 years. Many operations, including stripmap, spotlight, scan, and multiple platform-borne SAR systems have become more and more popular in recent years. SAR systems have been used in many fields, such as soil moisture, forestry, wetland, and agriculture. Due to the higher resolution of SAR image required, the accuracies of the echo models and imaging algorithms need improvement. Because the echo model is a kind of output, it forms a theoretical basis for all SAR imaging algorithms. From an engineering point of view, the traditional echo model is the time-delayed signal of the transmitted signal. There are lots of approximations for echo model. Let us recall the echo signal model. The SAR echo is one of electromagnetic wave forms, and Maxwell's equations are the basic and accurate tools for electromagnetic wave measurement. Mathematically, the SAR imaging procedure is an inverse problem of the electromagnetic wave. Many mathematical and practical researchers are interested in these types of inverse problems [1-5].

In recent studies of SAR and inverse synthetic aperture radar (ISAR), the data size trends larger and larger as

\footnotetext{
*Correspondence: chenjie@buaa.edu.cn

1 School of Electronics and Information Engineering, Beihang University, 37

Xueyuan Road, Haidian District, Beijing 100191, China

Full list of author information is available at the end of the article
}

the need for high resolution images becomes greater and greater. It costs too much and many times, the data cannot be downloaded in real time from some space-borne platforms, such as satellites. How to disperse the data size efficiently and decrease the data ratio is a real problem for engineers. The good news is that compressive sensing (CS) theory addresses at least some of these problems [6]. Random sampling theory, the CS sampling, and construction proposed by Donoho et al. in 2006 [7-9] and Baraniuk in 2007 [10] may also help in this endeavor. Many publications in the literature cast the CS into radar imaging [11-16], where they analyzed the sparse characteristics of SAR signals in different domains. Those are the fundamentals of reconstructing the scenes from the equivalent down-sampling data set. Another application of the CS SAR imaging is to construct the CS matrix and its optimization [17]. However, most of the past research works are focused on the classical echo model for SAR [18] and ISAR image [19].

In this paper, our goal is to analyze a general echo model and construct a new CS matrix for the SAR imaging. We use the partial differential Maxwell's equations to derive the electronic field and then obtain the general echo model in frequency domain and time domain. Based on this general model, considering the sparse characteristic of the scene, we will construct the corresponding orthogonal CS matrix for the SAR imaging.

\section{Springer}

(c) 2014 Sun et al: licensee Springer. This is an Open Access article distributed under the terms of the Creative Commons Attribution License (http://creativecommons.org/licenses/by/4.0), which permits unrestricted use, distribution, and reproduction in any medium, provided the original work is properly credited. 
Our paper is organized as follows. Section 2 derives the general SAR echo model based on Maxwell's equations. In Section 3, a new CS matrix is constructed for the CS imaging based on the above general echo model in frequency domain. In Section 4, simulations are given to validate the orthogonality of the CS matrix and the imaging performance. Some comparisons of two CS methods and the evaluation method with indices are also provided. Section 5 concludes the paper.

\section{General SAR echo model}

From the scalar form of Maxwell's equations, the incident and scattering fields are analyzed first, and then the general SAR echo model [20] is derived according to the antenna theory.

\subsection{Maxwell's equations and field expressions}

In this paper, the simplified scalar form of Maxwell's equations is used directly instead of the vector form of Maxwell's equations [21]. That is,

$$
\left(\nabla^{2}-c^{-2}(\mathbf{x}) \partial_{t}^{2}\right) \varepsilon^{\text {tot }}(t, \mathbf{x})=-j(t, \mathbf{x})
$$

where $\mathbf{x}$ is the three-dimensional position vector, $c(\mathbf{x})$ is the local propagation speed of electromagnetic waves and $c(\mathbf{x})=c_{0}$ in free space (usually, $c_{0}$ is the speed of light), $\varepsilon^{\text {tot }}(t, \mathbf{x})$ and $j(t, \mathbf{x})$ is the total scalar field and the current density on the antenna, respectively. $c(\mathbf{x})$ satisfies $c^{-2}(\mathbf{x})=c_{0}^{-2}-V(\mathbf{x})$, where $V(\mathbf{x})$ stands for the target reflectivity function, which will be reconstructed from radar echoes. $\varepsilon^{\text {tot }}(t, \mathbf{x})=\varepsilon^{\text {in }}(t, \mathbf{x})+\varepsilon^{\text {sc }}(t, \mathbf{x})$, where $\varepsilon^{\text {in }}(t, \mathbf{x})$ and $\varepsilon^{\mathrm{sc}}(t, \mathbf{x})$ are the incident scalar field and the scattered scalar field, respectively. And $\varepsilon^{\text {in }}(t, \mathbf{x})$ satisfies

$$
\left(\nabla^{2}-c_{0}^{-2} \partial_{t}^{2}\right) \varepsilon^{\text {in }}(t, \mathbf{x})=-j(t, \mathbf{x}) .
$$

Then, the expressions of scattered field and the incident field are as follows:

$$
\begin{gathered}
\varepsilon^{\mathrm{sc}}(t, \mathbf{x})=\iint g(t-\tau, \mathbf{x}-\mathbf{z}) V(\mathbf{z}) \partial_{\tau}^{2} \varepsilon^{\mathrm{tot}}(\tau, \mathbf{x}) d \tau d \mathbf{z} \\
\varepsilon^{\text {in }}(t, \mathbf{x})=-\iint g(t-\tau, \mathbf{x}-\mathbf{z}) j(\tau, \mathbf{x}) d \tau d \mathbf{z}
\end{gathered}
$$

where $g(t, \mathbf{x})=\frac{\delta\left(t-|\mathbf{x}| / c_{0}\right)}{4 \pi|\mathbf{x}|}$, called Green's function [22], is the fundamental solution of the partial differential equation $\left(\nabla^{2}-c_{0}^{-2} \partial_{t}^{2}\right) g(t, \mathbf{x})=-\delta(t) \delta(\mathbf{x})$.
Considering the single scattering approximation in Equation 3, the scattered field is reduced to

$$
\varepsilon^{\mathrm{sc}}(t, \mathbf{x}) \approx \iint g(t-\tau, \mathbf{x}-\mathbf{z}) V(\mathbf{z}) \partial_{\tau}^{2} \varepsilon^{\mathrm{in}}(\tau, \mathbf{x}) d \tau d \mathbf{z}
$$

For simplicity, the following analysis is complemented in frequency domain $\omega$ instead of time domain $t$.

$$
\begin{aligned}
& E_{B}^{\mathrm{sc}}(\omega, \mathbf{x})=-\int G(\omega, \mathbf{x}) V(\mathbf{z}) \omega^{2} E^{\mathrm{in}}(\omega, \mathbf{x}) d \mathbf{z} \\
& E^{\text {in }}(\omega, \mathbf{x})=\int G(\omega, \mathbf{x}-\mathbf{y}) J(\omega, \mathbf{y}) d \mathbf{y}
\end{aligned}
$$

where $E^{\text {sc }}(\omega, \mathbf{x})$ and $E^{\text {in }}(\omega, \mathbf{x})$ are the Fourier transforms of $\varepsilon^{\text {in }}(t, \mathbf{x})$ and $\varepsilon^{\text {in }}(t, \mathbf{x})$, respectively; $G(\omega, \mathbf{x})=\frac{e^{-i k|\mathbf{x}|}}{4 \pi|\mathbf{x}|}$ is the frequency expression of Green's function $g(t, \mathbf{x})$; $k=\frac{\omega}{c}$ is the wavenumber in range direction; and $J(\omega, \mathbf{x})$ is the current source in the frequency domain. Then, the scattered field with a theoretical point antenna is

$$
\begin{aligned}
E_{B}^{\mathrm{sc}}(\omega, \mathbf{x})= & -\iint G(\omega, \mathbf{x}-\mathbf{z}) G(\omega, \mathbf{x}-\mathbf{y}) \\
& \times V(\mathbf{z}) \omega^{2} J(\omega, \mathbf{y}) d \mathbf{y} d \mathbf{z}
\end{aligned}
$$

\subsection{Mathematical signal model}

\subsubsection{Radiation pattern for a SAR antenna}

Generally, the transmitting and receiving antenna is composed with many cells. For the sake of simplicity, a planar radar antenna over an aperture $[-a, a] \times[-b, b]$ is analyzed and the current density $I$ is constant; then, the radiation scalar $F(k, \mathbf{x})$ can be expressed by

$$
\begin{aligned}
F(k, \mathbf{x}) & =\int_{-a}^{a} \int_{-b}^{b} e^{i k \hat{\mathbf{x}} \cdot\left(s_{1} \hat{e}_{1}+s_{2} \hat{e}_{2}\right)} I d s_{1} d s_{2} \\
& =I\left(2 a \operatorname{sinc}\left(k a \hat{\mathbf{x}} \cdot \hat{e}_{2}\right)\right)\left(2 b \operatorname{sinc}\left(k b \hat{\mathbf{x}} \cdot \hat{e}_{1}\right)\right)
\end{aligned}
$$

where $\operatorname{sinc}(x)=\frac{\sin (x)}{x}, \hat{e}=\left(\hat{e}_{1}, \hat{e}_{2}\right)$ is corresponding to the antenna direction.

Let $p(t)$ be the transmitted signal; then, the current density on antenna $j(t, \mathbf{x})$ is proportional to $p(t)$ and independent of position. So, $J(\omega, \mathbf{x})$ is proportional to the spectrum of transmitted signal $P(\omega)$. Then,

$$
\begin{aligned}
F(k, \mathbf{x}) & =P(\omega)\left(2 a \operatorname{sinc}\left(k a \hat{\mathbf{x}} \cdot \hat{e}_{2}\right)\right)\left(2 b \operatorname{sinc}\left(k b \hat{\mathbf{x}} \cdot \hat{e}_{1}\right)\right) \\
& =P(\omega) G_{a}(k, \hat{\mathbf{x}}, \hat{e})
\end{aligned}
$$

where $G_{a}(k, \hat{\mathbf{x}}, \hat{e})$ is just an amplitude function independent of the transmitted signal, with a function of wavenumber $k$, which varies for a wideband signal even within a short pulse time duration. In fact, this corresponds to the frequency characteristics of antenna, especially under wide bandwidth. 


\subsubsection{Mathematical model of received echo}

Suppose that the center of antenna is located at $\mathbf{x}_{\mathbf{0}}$, the incident field $E^{\text {in }}(\omega, \mathbf{x})$ and scattered field $E_{B}^{\mathrm{sc}}(\omega, \mathbf{x})$ are

$$
\begin{aligned}
E^{\text {in }}(\omega, \mathbf{x})= & \int_{\mathbf{y} \in \text { antenna }} \frac{e^{-i k|\mathbf{x}-\mathbf{y}|}}{4 \pi|\mathbf{x}-\mathbf{y}|} P(\omega) d \mathbf{y} \\
\approx & \frac{e^{-i k\left|\mathbf{x}-\mathbf{x}_{\mathbf{0}}\right|}}{4 \pi\left|\mathbf{x}-\mathbf{x}_{\mathbf{0}}\right|} F\left(\widehat{k, \widehat{\mathbf{x}-\mathbf{x}_{\mathbf{0}}}}\right) \\
E_{B}^{\mathrm{sc}}(\omega, \mathbf{x}) \approx & -\int_{\mathbf{z} \in \operatorname{target}} \frac{e^{-i k|\mathbf{x}-\mathbf{z}|}}{4 \pi|\mathbf{x}-\mathbf{z}|} V(\mathbf{z}) \\
& \times \omega^{2} \frac{e^{-i k\left|\mathbf{z}-\mathbf{x}_{\mathbf{0}}\right|}}{4 \pi\left|\mathbf{z}-\mathbf{x}_{\mathbf{0}}\right|} F\left(\widehat{k} \widehat{\mathbf{x - \mathbf { x } _ { 0 }}}\right) d \mathbf{z} .
\end{aligned}
$$

When a monostatic SAR receives echoes, the stop-go approximation is applied and therefore the received echo is expressed by

$$
S_{\mathrm{rec}}(\omega)=\int_{\mathbf{y} \in \text { antenna }} E_{B}^{\mathrm{sc}}(\omega, \mathbf{y}) \mathrm{W}(\omega, \mathbf{y}) d \mathbf{y}
$$

where $\mathrm{W}(\omega, \mathbf{y})$ is the weight function of the antenna cell at the position y. Substitute Equation 12 into Equation 13, we have

$$
\begin{aligned}
D_{\text {rec }}\left(\omega ; \mathbf{x}_{0}\right) \approx & -\int_{\mathbf{z} \in \operatorname{target}}\left[\int_{\mathbf{y} \in \text { antenna }} \frac{e^{-i k|\mathbf{y}-\mathbf{z}|}}{4 \pi|\mathbf{y}-\mathbf{z}|} W(\omega, \mathbf{y}) d \mathbf{y}\right] \\
& \frac{V(\mathbf{z}) \omega^{2} e^{-i k\left|\mathbf{z}-\mathbf{x}_{0}\right|}}{4 \pi\left|\mathbf{z}-\mathbf{x}_{\mathbf{0}}\right|} F\left(k, \widehat{\mathbf{z}-\mathbf{x}_{\mathbf{0}}}\right) d \mathbf{z}
\end{aligned}
$$

Considering the far field condition, $|\mathbf{y}-\mathbf{z}| \approx\left|\mathbf{z}-\mathbf{x}_{\mathbf{0}}\right|-$ $\left(\widehat{\mathbf{z}-\mathbf{x}_{\mathbf{0}}}\right) \cdot\left(\mathbf{y}-\mathbf{x}_{\mathbf{0}}\right)$ and $|\mathbf{y}-\mathbf{z}|^{-1} \approx\left|\mathbf{z}-\mathbf{x}_{0}\right|^{-1}$ are adopted to produce the following form

$$
\begin{aligned}
D_{\text {rec }}\left(\omega ; \mathbf{x}_{0}\right) \approx & -\int_{\mathbf{z} \in \operatorname{target}}\left[\int_{\mathbf{y} \in \text { antenna }} e^{i k\left(\widehat{\mathbf{z - \mathbf { x } _ { 0 }}}\right) \cdot\left(\mathbf{y}-\mathbf{x}_{\mathbf{0}}\right)} W(\omega, \mathbf{y}) d \mathbf{y}\right] \\
& \times \frac{V(\mathbf{z}) \omega^{2} e^{-i 2 k\left|\mathbf{z}-\mathbf{x}_{\mathbf{0}}\right|}}{4 \pi\left|\mathbf{z}-\mathbf{x}_{\mathbf{0}}\right|^{2}} F\left(\widehat{\mathbf{z}-\mathbf{x}_{\mathbf{0}}}\right) d \mathbf{z}
\end{aligned}
$$

Putting the unit weight function $W(\omega, \mathbf{y})=1$ yields

$$
\begin{aligned}
D_{\mathrm{rec}}\left(\omega ; \mathbf{x}_{0}\right) \approx & -\int_{\mathbf{z} \in \operatorname{target}} V(\mathbf{z}) \omega^{2} P(\omega) \frac{e^{-i 2 k\left|\mathbf{z}-\mathbf{x}_{\mathbf{0}}\right|}}{\left(4 \pi\left|\mathbf{z}-\mathbf{x}_{\mathbf{0}}\right|\right)^{2}} \\
& \times G_{a}^{2}\left(k, \widehat{\mathbf{z}-\mathbf{x}_{\mathbf{0}}}, \hat{e}\right) d \mathbf{z} .
\end{aligned}
$$

Until now, we get the general echo model in frequency domain. According to the properties of Fourier transform, the general echo model in time domain can be expressed by

$$
\begin{aligned}
d_{\text {rec }}\left(t ; \mathbf{x}_{0}\right) \approx & \int_{\mathbf{z} \in \operatorname{target}} V(\mathbf{z}) \frac{\ddot{p}\left(t-2\left|\mathbf{z}-\mathbf{x}_{\mathbf{0}}\right| / c\right)}{\left(4 \pi\left|\mathbf{z}-\mathbf{x}_{\mathbf{0}}\right|\right)^{2}} \\
& \otimes g_{a}\left(t, \widehat{\mathbf{z - \mathbf { x } _ { 0 }}}, \hat{e}\right) d \mathbf{z}
\end{aligned}
$$

where $\ddot{p}(t)$ is the second derivative of $p(t), g_{a}\left(t, \widehat{\mathbf{z}-\mathbf{x}_{\mathbf{0}}}, \hat{e}\right)$ is the inverse Fourier transform of $G_{a}^{2}\left(k, \widehat{\mathbf{z - \mathbf { x } _ { \mathbf { 0 } }}}, \hat{e}\right)$, and $\otimes$ is convolution on $t$. If we neglect the antenna's variety with frequency $\omega$ or wavenumber $k$, that's $G_{a}(k, \hat{\mathbf{x}}, \hat{e}) \approx$ $G_{a}\left(k_{0}, \hat{\mathbf{x}}, \hat{e}\right)$, where $k_{0}=\frac{\omega_{0}}{c}$ is the wavenumber corresponding to the carrier frequency, the above expression can be simplified by

$$
\begin{aligned}
d_{\text {rec }}\left(t ; \mathbf{x}_{0}\right) \approx & \int_{\mathbf{z} \in \operatorname{target}} V(\mathbf{z}) \frac{\ddot{p}\left(t-2\left|\mathbf{z}-\mathbf{x}_{\mathbf{0}}\right| / c\right)}{\left(4 \pi\left|\mathbf{z}-\mathbf{x}_{\mathbf{0}}\right|\right)^{2}} \\
& \times G_{a}^{2}\left(k_{0}, \widehat{\mathbf{z}-\mathbf{x}_{\mathbf{0}}}, \hat{e}\right) d \mathbf{z}
\end{aligned}
$$

Even if some approximation about antenna radiation has been made, we can also find the difference between the above model and the transitional echo model, which is as simple as the summing the delayed signal of the transmitted signal. That is, the general echo is not the direct delay of the transmitted signal but the second order differential function of the transmitted signal. However, because Doppler phase is much more important in SAR imaging than the complex amplitude, the amplitude modulation is not serious for traditional SAR systems. In [20], the relation and difference between the general echo model and the classical model were minutely analyzed. However, for some high resolution applications, the differences may not be ignored. In this paper, to avoid error as much as possible, we try to find a CS matrix for the general echo model in frequency domain directly.

\section{Compressive sensing imaging for SAR}

\subsection{Basics of compressive sensing}

Because of the potential advantages for SAR imaging, there has been a wealth of research work directed towards CS since 2006. The spirit of CS is the sparse presentation under some basis. Suppose $\Psi \in \mathbf{C}^{N \times N}$ (in fact, the matrix was defined on $\mathbf{R}^{N \times N}$ originally and could be expanded to the complex matrix) is the orthogonal basis matrix and $\mathbf{s} \in \mathbf{R}^{N}$ is the coefficients vector, the signal $\mathbf{x} \in \mathbf{R}^{N}$ can be expressed by

$$
\mathbf{x}=\Psi \mathbf{s}
$$

where the transform coefficient $\mathbf{s}$ can be calculated by $\mathbf{s}=$ $\Psi^{-1} \mathbf{x}$ mathematically. If there are only $K(\ll N)$ non-zero values (or small absolution) in $\mathbf{s}$, the signal $\mathbf{x}$ is sparse in the corresponding domain and can be reconstructed by a few random samples with very high probability. Suppose the linear observing process is $\Phi \in \mathbf{R}^{M \times N}$, where $M<N$, the observation data $\mathbf{y} \in \mathbf{R}^{M}$ is

$$
\mathbf{y}=\Phi \mathbf{x}=\Phi \Psi \mathbf{s}=\Theta \mathbf{s}
$$


where the observing matrix $\Theta=\Phi \Psi \in \mathbf{C}^{M \times N}$. Based on CS theory, the reconstruction of the sparse coefficient $\mathbf{s}$ can be resolved by the following optimization problem.

$$
\hat{\mathbf{s}}=\arg \min \|\mathbf{s}\|_{0} \text { s.t. } \mathbf{y}=\Theta \mathbf{s}
$$

Because $l_{0}$ normalization optimization problem is difficult to resolve, $l_{0}$ normalization is replaced by $l_{1}$ normalization for the actual solution. Then, the signal $\mathbf{x}$ is to be estimated by $\hat{\mathbf{x}}=\Psi \hat{\mathbf{s}}$.

\subsection{Compressive sensing matrix for SAR imaging}

To apply CS to SAR imaging, we should consider two conditions: the sparsity of signal and the CS matrix. For the first condition, according to the theory of electromagnetic scattering, some targets can be thought as combinations of several strong scatterers; even for some continuous distributed scenes, the sparse coefficients can be found in the frequency domain or wavelet domain, etc. So, the sparsity of echo signal has been discussed for some special applications, such as ocean remote sensing. For the other condition, the previous literature $[23,24]$ presented one kind of sensing matrices in time domain as follows.

$\Psi=\left[\begin{array}{ccccc}\operatorname{chirp}\left(\frac{N_{\tau}}{2}\right) & \operatorname{chirp}\left(\frac{N_{\tau}}{2}-1\right) & \operatorname{chirp}\left(\frac{N_{\tau}}{2}-2\right) & \ldots & 0 \\ \operatorname{chirp}\left(\frac{N_{\tau}}{2}+1\right) & \operatorname{chirp}\left(\frac{N_{\tau}}{2}\right) & \operatorname{chirp}\left(\frac{N_{\tau}}{2}-1\right) & \ldots & 0 \\ \operatorname{chirp}\left(\frac{N_{\tau}}{2}+2\right) & \operatorname{chirp}\left(\frac{N_{\tau}}{2}+1\right) & \operatorname{chirp}\left(\frac{N_{\tau}}{2}\right) & \ldots & 0 \\ \vdots & \vdots & \vdots & \ddots & \vdots \\ 0 & 0 & 0 & \ldots & \operatorname{chirp}\left(\frac{N_{\tau}}{2}\right)\end{array}\right]$

where $\operatorname{chirp}(i)=\operatorname{rect}\left(\frac{2 i-N_{\tau}}{2 N_{\tau}}\right) \exp \left\{j \pi b\left(\frac{i-0.5 * N_{\tau}}{F s}\right)^{2}\right\}$ is the transmitted linear frequency modulation (LFM) chirp signal, $b$ is the frequency modulation ratio of the chirp signal, $f_{s}$ is the sampling frequency, and $\tau$ and $N_{\tau}=\tau f_{s}$ are the pulse width and point numbers of the chirp signal, respectively.

Table 1 Main simulation parameters

\begin{tabular}{lc}
\hline Parameter & Value \\
\hline Height of antenna & $5000 \mathrm{~m}$ \\
Velocity of antenna & $200 \mathrm{~m} / \mathrm{s}$ \\
Look angle & $45^{\circ}$ \\
Frequency of carrier & $1 \mathrm{GHz}$ \\
Bandwidth of chirp signal & $400 \mathrm{MHz}$ \\
Sampling frequency & $500 \mathrm{MHz}$ \\
Pulse width of chirp signal & $1 \mathrm{Hs}$ \\
Pulse repetition frequency & $500 \mathrm{~Hz}$ \\
Number of range cells & 1,024 \\
Number of down-sampled range cells & 256 \\
Synthetic aperture time & $5 \mathrm{~s}$ \\
\hline
\end{tabular}

This CS matrix is based on the idea that the echo signal is the delay of the transmitted signal, and its orthogonality also was validated. However, according to Section 2 of this paper, we have found that the general echo model is the expansion of the simple delay model of the transmitted signal, and it is more accurate for some high resolution applications. Our goal is to find a CS matrix corresponding to the general echo model directly.

In the beginning, the received signal after discrete processing from the frequency domain expression (Equation 16) can be expressed as

$$
\begin{aligned}
D_{\text {rec }}\left(m, n ; \mathbf{x}_{\mathbf{0}}\right)= & -\sum_{n=1}^{N_{p}} \sigma_{n} \omega_{m}^{2} P\left(\omega_{m}\right) \frac{e^{-i 4 \omega_{m} R_{n} / c}}{\left(4 \pi R_{n}\right)^{2}} \\
& \times G_{a}^{2}\left(\frac{\omega_{m}}{c}, \widehat{\mathbf{z}_{\mathbf{n}}-\mathbf{x}_{\mathbf{0}}}, \hat{e}\right)
\end{aligned}
$$

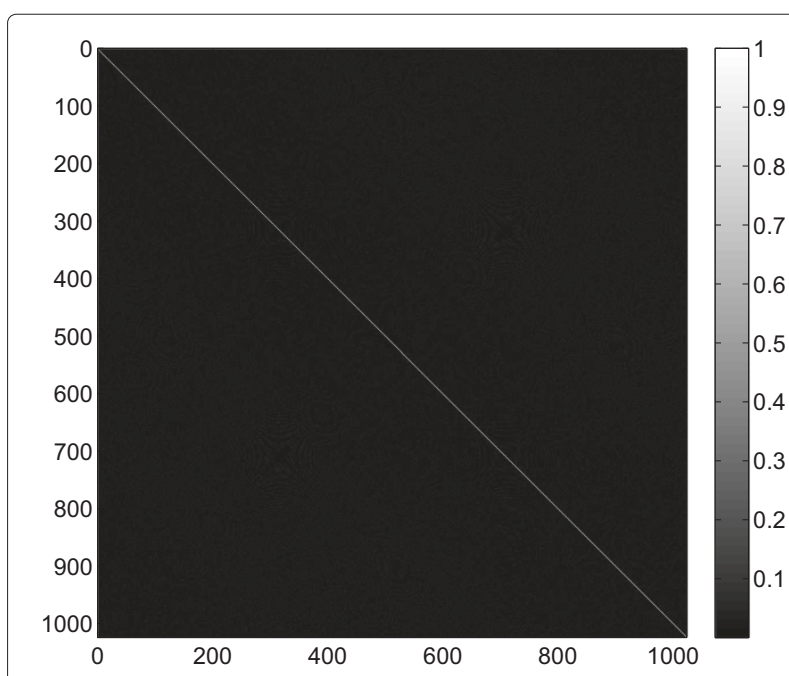

(a)

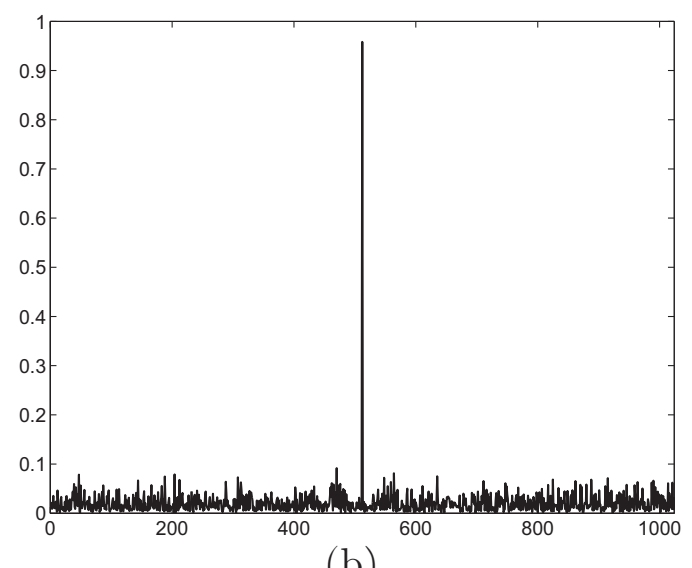

(b)

Figure 1 Correlation matrix of the CS matrix. (a) The two dimensional distribution of the square correlation matrix; (b) the profile selected from the square matrix along with the 512th row. 
where the subscript $m$ represents the frequency number for fast time of echo, $n$ represents the target index, $N_{p}$ is the number of the discrete targets, $R_{n}$ is the range between the $n$th target point located at $\mathbf{z}_{n}$ and the center of the antenna located at $\mathbf{x}_{0}, \omega_{m}=2 \pi f_{s}(m / N-1 / 2)$ is the discrete frequency, $m=0,1, \ldots, N-1$, and $N$ is the number of Fourier transform. Suppose the scatter coefficients vector $\Sigma=\left[\sigma_{1}, \sigma_{2}, \ldots, \sigma_{N_{p}}\right]^{T}$, the $(m, n)$-th element of the CS matrix $\Psi$ can be constructed as

$$
\Psi_{m n}=\omega_{m}^{2} P\left(\omega_{m}\right) \frac{e^{-i 4 \omega_{m} R_{n} / c}}{\left(4 \pi R_{n}\right)^{2}} G_{a}^{2}\left(\frac{\omega_{m}}{c}, \widehat{\mathbf{z}_{\mathbf{n}}-\mathbf{x}_{\mathbf{0}}}, \hat{e}\right)
$$

With these definitions, it is not hard to validate $\mathbf{D}_{\text {rec }}\left(\mathbf{x}_{\mathbf{0}}\right)=\Psi \Sigma$. Because we use the general echo model in frequency domain, the CS matrix $\Psi$ is also the function of the general transmitted signal in frequency domain $P\left(\omega_{m}\right)$. For instance, if the transmitted signal is a chirp signal, according to the principle of stationary phase, we can get $P\left(\omega_{m}\right)=A e^{-i\left(\frac{\omega_{n}^{2}}{4 \pi b}+\frac{\pi}{4}\right)}$, where $A$ is a complex constant, and then the CS matrix $\Psi$ becomes

$$
\Psi_{m n}=\frac{A \omega_{m}^{2} e^{-i \frac{\pi}{4}}}{\left(4 \pi R_{n}\right)^{2}} e^{-i\left(\frac{4 \omega_{m} R_{n}}{c}+\frac{\omega_{n}^{2}}{4 \pi b}\right)} G_{a}^{2}\left(\frac{\omega_{m}}{c}, \widehat{\mathbf{z}_{\mathbf{n}}-\mathbf{x}_{\mathbf{0}}}, \hat{e}\right)
$$

To validate the orthogonality of the CS matrix for different rows, the following correlation will be calculated

$$
\operatorname{Cor}\left(\Psi_{m_{1} n}, \Psi_{m_{2} n}\right)=\sum_{n=1}^{N} \Psi_{m_{1} n} \Psi_{m_{2} n}^{*} m_{1}, m_{2} \in[1, N]
$$

It is not hard to find that $\left|\operatorname{Cor}\left(\Psi_{m_{1} n}, \Psi_{m_{1} n}\right)\right| \gg$ $\left|\operatorname{Cor}\left(\Psi_{m_{1} n}, \Psi_{m_{2} n}\right)\right|,\left(m_{1} \neq m_{2}\right)$ because of the cophasal stacking effect. And after normalization for each row, the correlation matrix is approximately an identical matrix. The orthogonality will be validated in the next section. On the other hand, besides Gaussian and Bernoulli matrices, another very important class of structured random matrices is the random partial Fourier matrix, which is also the object of study in the very first paper on CS [9]. In fact, a random partial Fourier matrix relates the time domain signal and the sparse spectrum items; also, it is the first time to construct an orthonormal basis in $\mathrm{C}^{N \times N}$ rather than $\mathbf{R}^{N \times N}$. It has been proved that the Fourier matrix satisfied the restricted isometry property (RIP) and can be applied to CS reconstruction. The proposed matrix defined by Equations 24 or 25 can be thought as one form of the Fourier matrix and can be applied for CS imaging for the general SAR echo model. Randomly selected $M$ rows from $\Psi$ will generate a random partial Fourier matrix $\Theta$; the
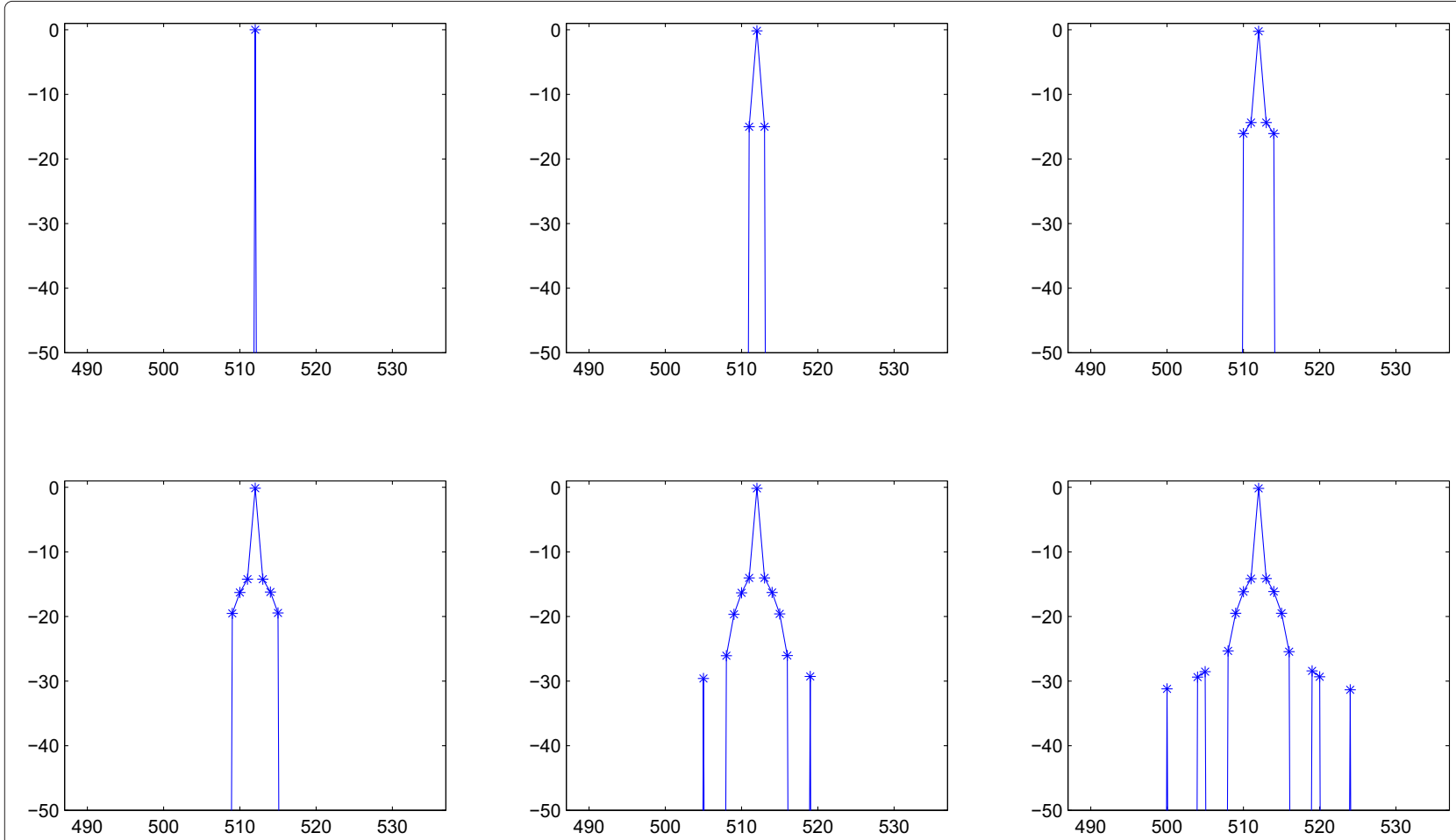

Figure 2 Non-zero points of reconstruction result corresponding to different $\boldsymbol{K}$ without interpolation. The six figures correspond to $K=1,3,5,7,11,15$ 


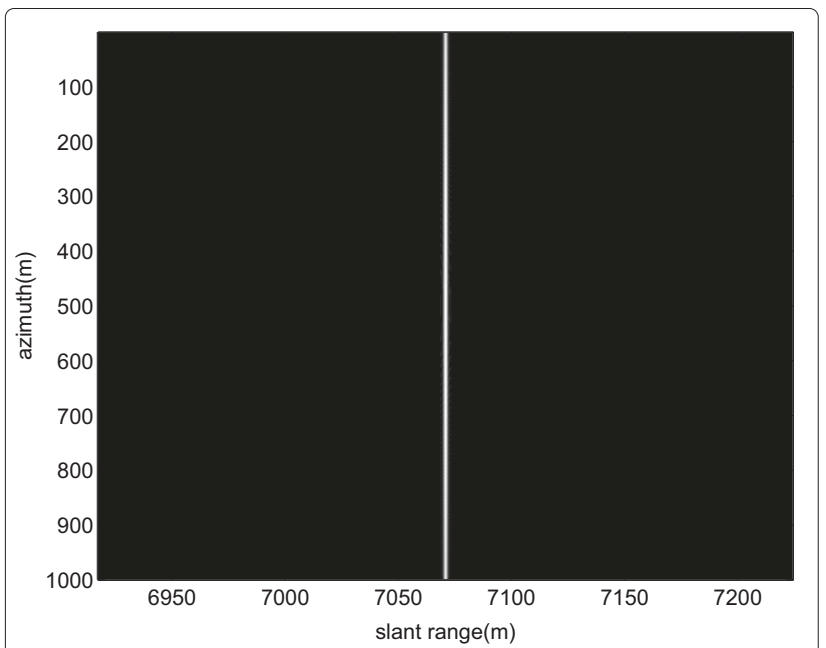

(a)

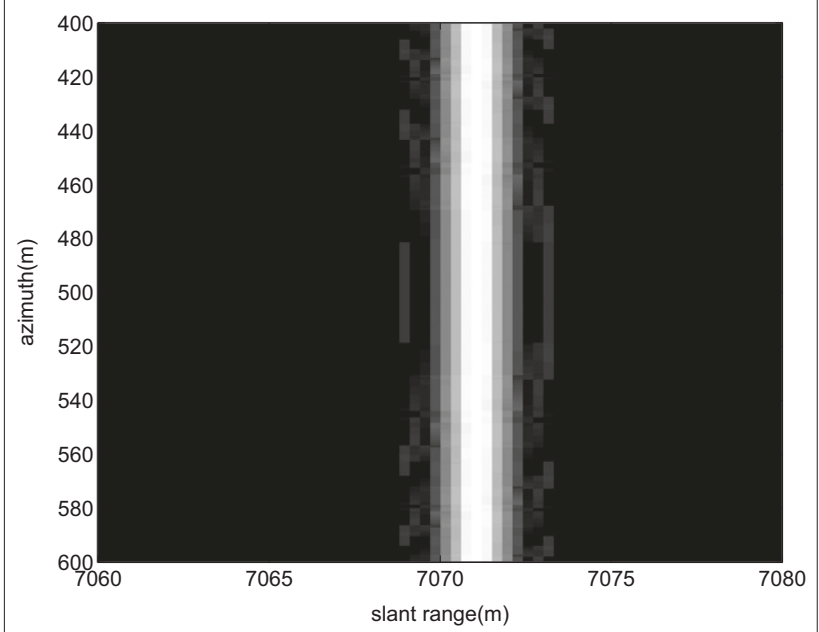

(b)

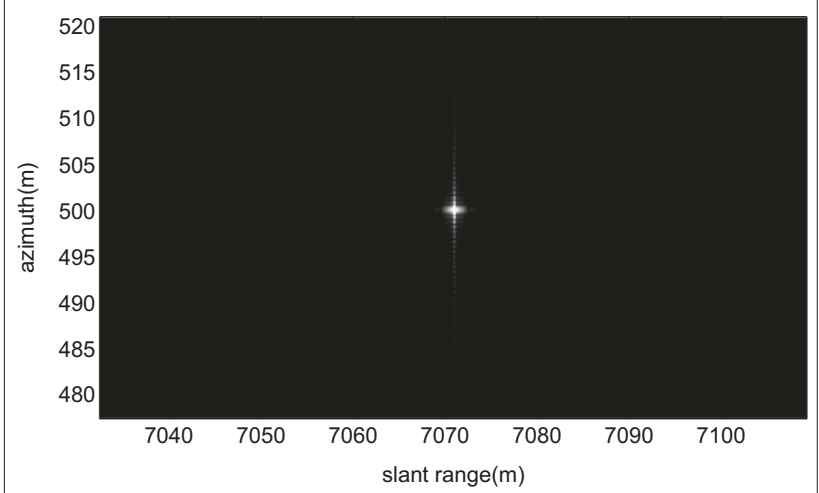

(c)

Figure 3 Simulation results based on our proposed matrix in the frequency domain. (a) Range CS compression after range cell migration correction; (b) zoom figure of (a); (c) image after azimuth processing. range signal reconstruction from the SAR echoes can be accomplished in range frequency domain instead of the traditional match filtering.

In this paper, the orthogonal match pursuit (OMP) algorithm [25] is unitized for the reconstruction of the sparse

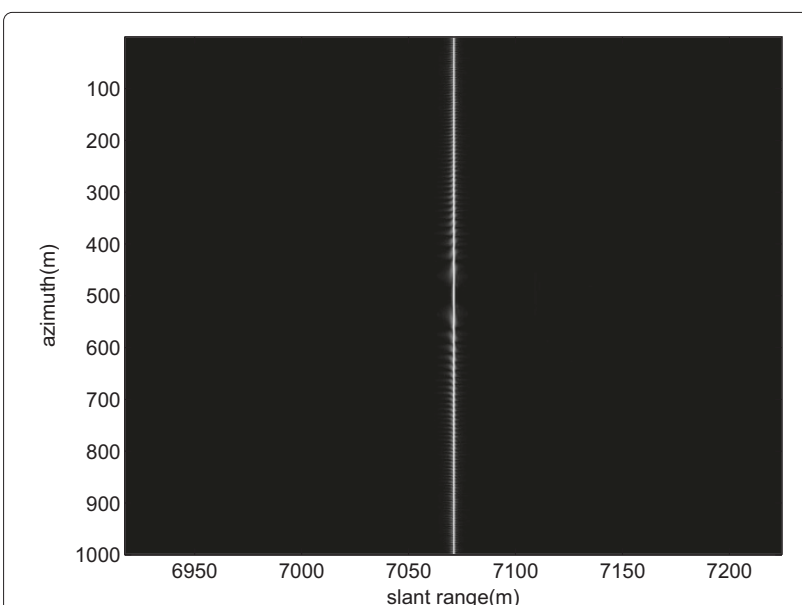

(a)

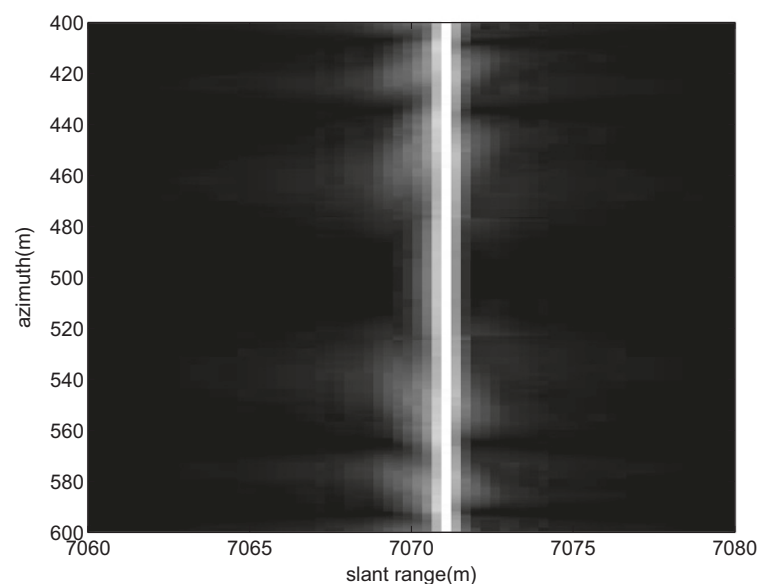

(b)

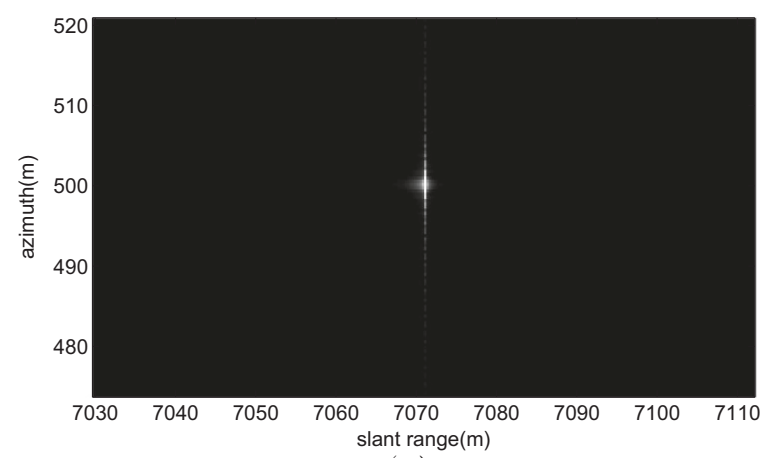

(c)

Figure 4 Simulation results based on the previous matrix in the time domain. (a) Range CS compression after range cell migration correction; (b) zoom figure of (a); (c) image after azimuth processing. 

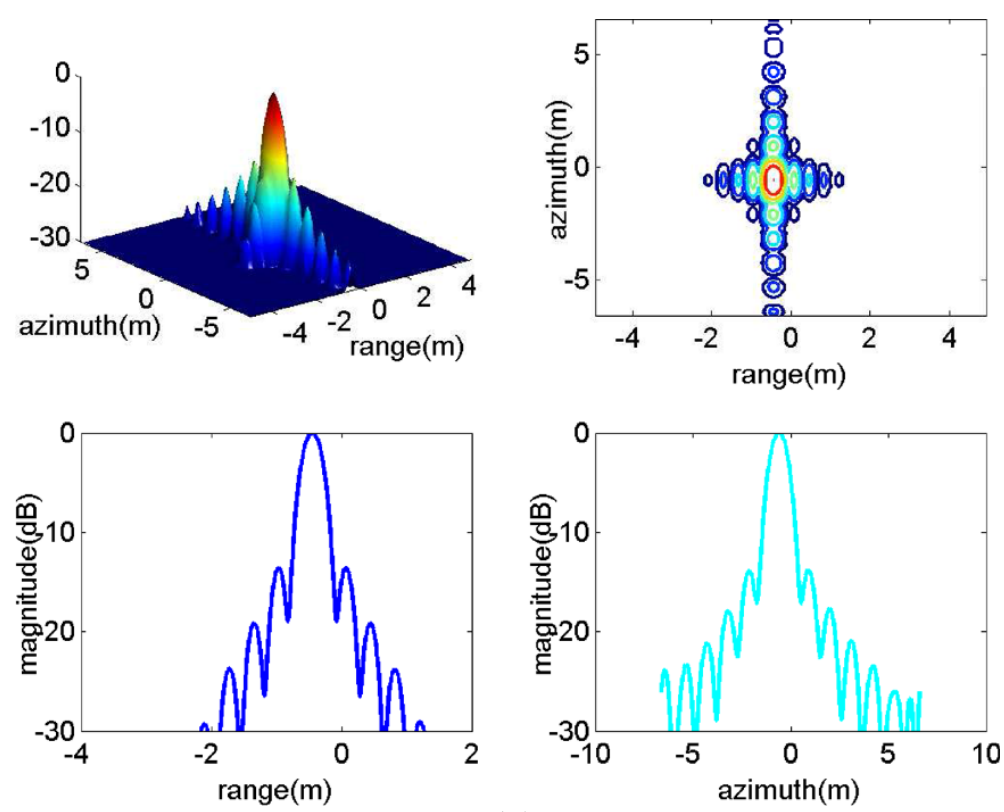

(a)
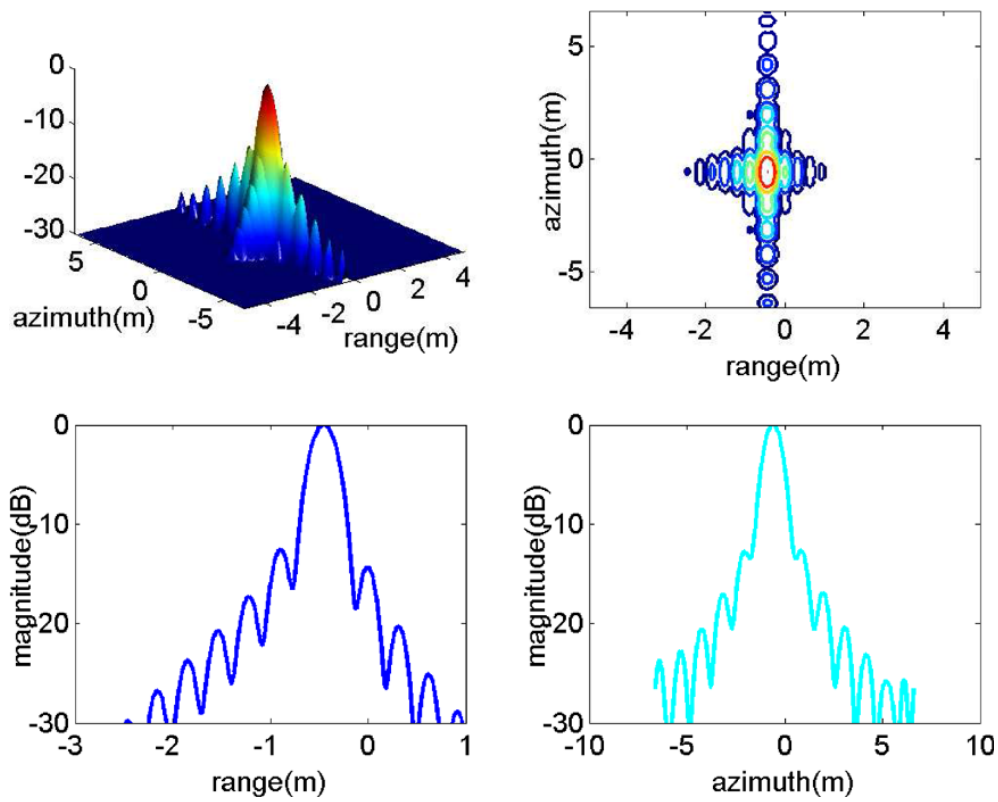

(b)

Figure 5 Point quality evaluation results. (a) Results based on our proposed matrix in frequency domain; (b) results based on the previous matrix in time domain. The four subfigures in both (a) and (b) stand the three-dimensional surface, contour image, range profile, and azimuth profile. The two-dimensional and one-dimensional Fourier interpolations are used, and the times of interpolation are 64 and 1,024.

Table 2 Point evaluation indices of two CS methods

\begin{tabular}{|c|c|c|c|c|}
\hline \multirow{2}{*}{ Index } & \multicolumn{2}{|c|}{ Slant range } & \multicolumn{2}{|c|}{ Azimuth } \\
\hline & Resolution (m) & PSLR (dB) & Resolution (m) & PSLR (dB) \\
\hline Results based on the previous matrix & 0.2860 & -12.60 & 1.0094 & -12.68 \\
\hline Results based on our proposed matrix & 0.3277 & -13.39 & 0.9867 & -13.79 \\
\hline Theoretical values & 0.3322 & -13.26 & 0.9397 & -13.26 \\
\hline
\end{tabular}


signal, which corresponds to the discrete scattering coefficients in SAR imaging. The orthogonality of the CS matrix makes sure of the maximum probability of reconstruction quickly.

\section{Simulations}

In this section, the properties of the CS matrix $\Psi$ are analyzed first, then the CS imaging for a point scene is simulated with both the previous time-domain CS matrix like Equation 22 and the proposed matrix corresponding to the general echo model defined by Equation 24. The comparison of the range reconstruction results after CS and the final imaging results are given.

\subsection{Simulation parameters}

The main parameters for the below simulations are listed in Table 1. To compare with the time-domain CS method, the most common chirp signal is chosen as the

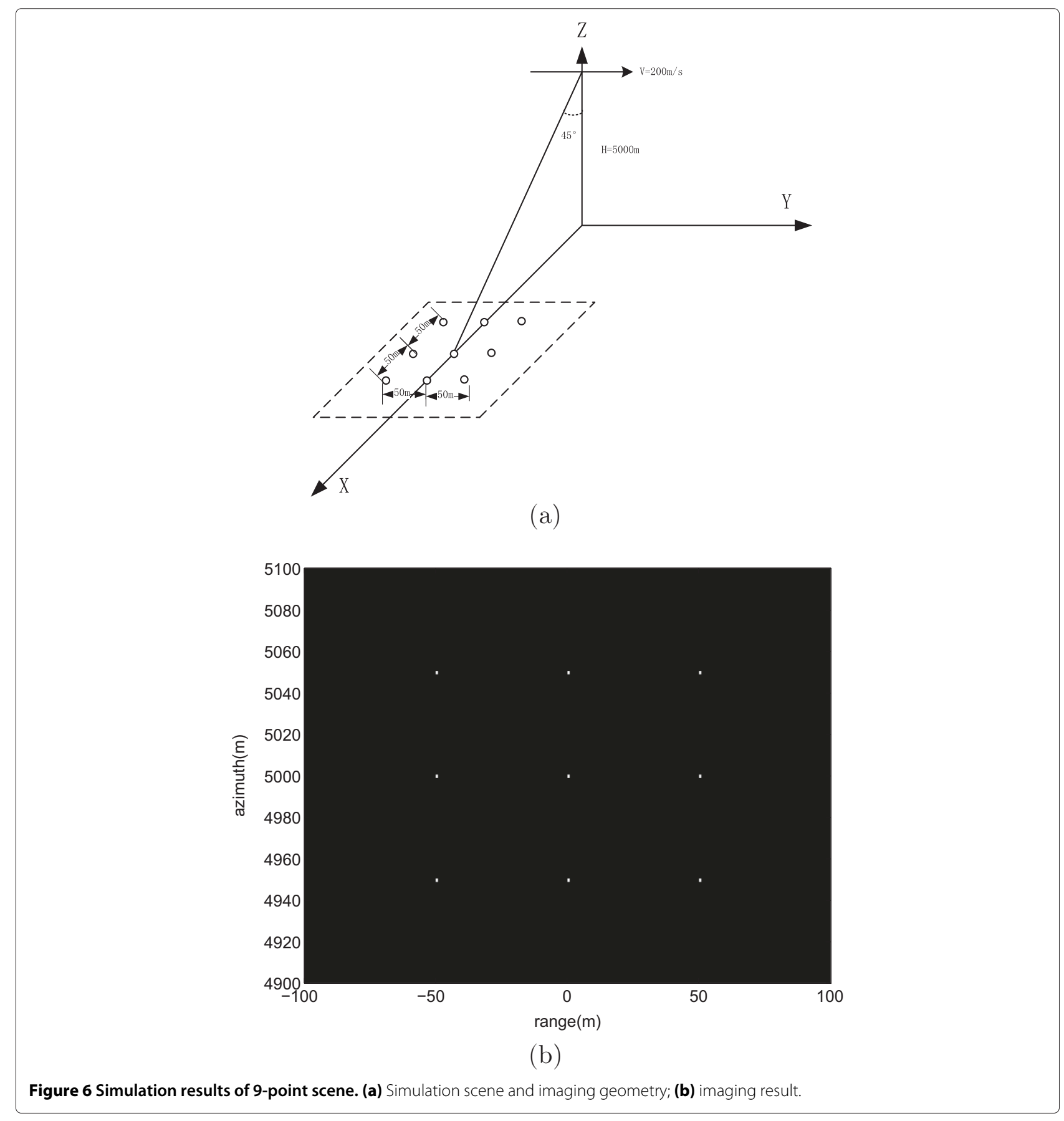


transmitted signal. In order to display the affections of wide bandwidth, the frequency of chirp signal ranges from 800 to $1,200 \mathrm{MHz}$, and the ratio of bandwidth and carrier frequency is up to $40 \%$.

\subsection{Results and discussion}

\subsubsection{Compressive sensing matrix properties}

Since there is some complex magnitude term in Equation 25, the accurate proof of the orthogonality based on Equation 26 is difficult. However, Figure 1 illustrates the correlation matrix of $\Psi$ by numerical simulation.

According to Figure 1, the diagonal elements of the correlation matrix trend to 1 , and others are very small. This correlation matrix is approximate to the identical matrix, so the CS matrix $\Psi$ can be thought as orthogonal matrix.

\subsubsection{Imaging results and evaluation}

Before analyzing the simulation results, we discuss the evaluation method first. Fourier interpolation is often applied into the evaluation of the traditional range compressed result or SAR image. However, in the previous CS reconstruction, it is acceptable that the range reconstruction result has no sidelobe $[18,26,27]$, and Fourier interpolation is not suitable to evaluate the result. This phenomenon might be explained that when $K$ is 1 or a very small number, after reconstruction of compressive sensing, there is at most only 1 non-zero value in each range profile, like a delta function, and any interpolation does not fit during evaluation. According to our simulation during range $\mathrm{CS}$ reconstruction, we still set different sparse coefficients $K$ for a single target, and a bigger $K$ will expose the more sidelobes.

Figure 2 illustrates the increasing process of non-zero points corresponding to different $K$ without interpolation. The bigger $K$ is, the more obvious the sidelobes are. For example, when $K=1$, there is only 1 non-zero point, which likes a delta function and when $K=15$, there are at least two pairs of sidelobes nearby the peak. Also Fourier interpolation can be carried out for this construction with some sidelobes. Meanwhile, we set $K=11$ when we apply the previous CS matrix to the same echoes.

To quantitatively analyze the effect of the CS imaging, Figures 3 and 4 show the corresponding results of range reconstruction and final imaging with two kinds of CS matrices.

According to the above results, it is easy to find that there are a few sidelobes in both range reconstructions in Figure $3 \mathrm{~b}$ and Figure $4 \mathrm{~b}$. The range reconstruction results based on our proposed method shown in Figure 3 are better than those based on the previous matrix shown in Figure 4. Also, it is easy to find the symmetry of the range result after $\mathrm{CS}$, and the final result by our matrix is better.
Moreover, we evaluate the imaging results with Fourier interpolation, shown in Figure 5. The resolutions and peak sidelobe ratios (PSLR) of both slant range and azimuth profiles are listed in Table 2.

According to Figure 5, the symmetry along with range direction of the result based on our proposed matrix is better than those based on the previous matrix. That is also the main reason of the slant range PSLR difference in Table 2. Also, the evaluation indices of our proposed method are much closer to the theoretical indices. Usually the higher the range resolution is, the better the image effect is. However, the more the indices approach the theoretical values, the better the reconstruction algorithm is. In this respect, the CS imaging based on our proposed matrix is much better.

Moreover, a 9-point scene was simulated. Figure 6 displayed the scene and imaging result by our method. Figure 6a shows the 9 points and the imaging geometry. Figure $6 \mathrm{~b}$ implies that the proposed method based on the CS matrix in the frequency domain is capable of imaging a small set of scatterers.

\section{Conclusions}

In this paper, the general echo model is derived from Maxwell's equations. The general echo expressions in both frequency domain and time domain are given after generating the scatter field. The general echo model is the expansion of the classical echo model. Based on the general echo model in the frequency domain, a new CS matrix like a random partial Fourier matrix is constructed to apply for the CS imaging. Simulation results validate the orthogonality of the proposed CS matrix and the indices of the CS imaging by our model approach the theoretical values better. Also a bigger sparse number $K$ will expose the sidelobes of the reconstruction, and Fourier interpolation can be applied into evaluating the imaging results.

\section{Competing interests \\ The authors declare that they have no competing interests.}

\section{Acknowledgements}

This work is supported by the National Natural Science Fund of China (Grant Nos. 61301187 and 61328103), and the Fundamental Research Funds for the Central Universities. ZQ thanks the U.S. Department of Education GAANN project (P200A120256) for supporting the UTPA mathematics graduate program. The authors also thank Mr. John Montalbo for his communication and the anonymous reviewers for their valuable suggestions.

\section{Author details \\ ${ }^{1}$ School of Electronics and Information Engineering, Beihang University, 37 Xueyuan Road, Haidian District, Beijing 100191, China. ${ }^{2}$ Department of Mathematics, the University of Texas-Pan American, 1201 West University Drive, Edinburg 78539, TX, USA. ${ }^{3}$ Mathematics Department, Washington State University, PO box 641227, Pullman 99163, WA, USA.}

Received: 27 February 2014 Accepted: 3 October 2014

Published: 10 October 2014 


\section{References}

1. M Soumekh, M Kaveh, A theoretical study of model approximation errors in diffraction tomography. IEEE Trans. Ultrason. Ferroelectrics Freq. Contr. 33(1), 10-20 (1986)

2. M Soumekh, A system model and inversion for synthetic aperture radar imaging. IEEE Trans. Image Process. 1(1), 64-76 (1992)

3. M Cheney, A mathematical tutorial on synthetic aperture radar. SIAM Rev. 43(2), 301-312 (2001)

4. CJ Nolan, M Cheney, Synthetic aperture inversion. Inverse. Probl. 18(1), 221-235 (2002)

5. JX Lopez, Z Qiao, Filtered back projection inversion of turntable ISAR data, in Proc. SPIE, vol. 8051, (2011), pp. 80510901-80510909. doi:10.1117/12.884419

6. J-J Fuchs, On the application of the global matched filter to doa estimation with uniform circular arrays. IEEE Trans. Signal Process. 49(4), 702-709 (2001)

7. EJ Candès, J Romberg, T Tao, Robust uncertainty principles: exact signal reconstruction from highly incomplete frequency information. IEEE Trans. Inform. Theor. 52(2), 489-509 (2006)

8. DL Donoho, Compressed sensing. IEEE Trans. Inform. Theor. 52(4), 1289-1306 (2006)

9. EJ Candes, T Tao, Near-optimal signal recovery from random projections: universal encoding strategies. IEEE Trans.Inform. Theor. 52(12), 5406-5425 (2006)

10. R Baraniuk, Compressive sensing. IEEE Signal Process. Mag. 24(4), 118-120124 (2007)

11. R Baraniuk, P Steeghs, Compressive radar imaging, in Radar Conference, 2007 IEEE (IEEE, 2007), pp. 128-133. doi:10.1109/RADAR.2007.374203

12. M Herman, T Strohmer, Compressed sensing radar, in Radar Conference, 2008. RADAR'08. IEEE (IEEE, 2008), pp. 1-6

13. JH Ender, On compressive sensing applied to radar. Signal Process. 90(5), 1402-1414 (2010)

14. LC Potter, E Ertin, JT Parker, M Cetin, Sparsity and compressed sensing in radar imaging. Proc. IEEE. 98(6), 1006-1020 (2010)

15. E Lagunas, MG Amin, F Ahmad, M Nájar, Sparsity-based radar imaging of building structures, in Signal Processing Conference (EUSIPCO), 2012 Proceedings of the 20th European (IEEE, 2012), pp. 864-868

16. LXu, Q Liang, X Cheng, D Chen, Compressive sensing in distributed radar sensor networks using pulse compression waveforms. EURASIP J. Wireless Commun. Netw. 2013(1), 1-10 (2013)

17. J Xu, Y Pi, Z Cao, Optimized projection matrix for compressive sensing. EURASIP J. Adv. Signal Process. 2010, 43 (2010)

18. Q Liang, Compressive sensing for synthetic aperture radar in fast-time and slow-time domains, in Signals, Systems and Computers (ASILOMAR), 2011 Conference Record of the Forty Fifth Asilomar Conference On, (2011), pp. 1479-1483. doi:10.1109/ACSSC.2011.6190264

19. L Zhang, M Xing, C-W Qiu, J Li, J Sheng, Y Li, Z Bao, Resolution enhancement for inversed synthetic aperture radar imaging under low snr via improved compressive sensing. IEEE Trans. Geosci. Rem. Sens. 48(10), 3824-3838 (2010)

20. B Sun, Z Qiao, J Chen, Outer circular synthetic apeture radar imaging based on maxwell's equations. J. Appl. Remote Sens. 6(1), $06354701-06354711$ (2012)

21. M Cheney, B Borden, Fundamentals of Radar Imaging, vol. 79. (Siam, California, 2009)

22. T Myint-U, L Debnath, Linear Partial Differential Equations for Scientists and Engineers. (Springer, Birkhäuser Boston, 2007)

23. M Tello Alonso, P López-Dekker, JJ Mallorquí, A novel strategy for radar imaging based on compressive sensing. IEEE Trans. Geosci. Rem. Sens. 48(12), 4285-4295 (2010)

24. $\mathrm{P}$ Xiao, $\mathrm{Z} \mathrm{Yu,} \mathrm{C} \mathrm{Li,} \mathrm{Compressive} \mathrm{sensing} \mathrm{sar} \mathrm{range} \mathrm{compression} \mathrm{with} \mathrm{chirp}$ scaling principle. Sci. China Inform. Sci. 55(10), 2292-2300 (2012)
25. JA Tropp, AC Gilbert, Signal recovery from random measurements via orthogonal matching pursuit. IEEE Trans. Inform. Theor. 53(12), 4655-4666 (2007)

26. J Xu, Y Pi, Compressive sensing in radar high resolution range imaging. J. Comput. Inform. Syst. 3, 778-785 (2011)

27. J Yang, J Thompson, X Huang, T Jin, Z Zhou, Segmented reconstruction for compressed sensing sar imaging. IEEE Trans. Geosci. Rem. Sens. 51(7), 4214-4225 (2013)

doi:10.1186/1687-6180-2014-153

Cite this article as: Sun et al:: Compressive sensing imaging for general synthetic aperture radar echo model based on Maxwell's equations. EURASIP Journal on Advances in Signal Processing 2014 2014:153.

\section{Submit your manuscript to a SpringerOpen ${ }^{\mathcal{O}}$ journal and benefit from:}

- Convenient online submission

- Rigorous peer review

- Immediate publication on acceptance

- Open access: articles freely available online

- High visibility within the field

- Retaining the copyright to your article

Submit your next manuscript at $\boldsymbol{\wedge}$ springeropen.com 